\title{
Simulating hemodynamics of the Fontan Y-graft based on patient-specific in vivo connections
}

\author{
Christopher M. Haggerty, BS, ${ }^{a}$ Kirk R. Kanter, MD, ${ }^{\mathrm{b}}$ Maria Restrepo, BS, ${ }^{\mathrm{a}}$ Diane A. de Zélicourt, PhD, ${ }^{\mathrm{a}}$ \\ W. James Parks, MD, ${ }^{\mathrm{c}}$ Jarek Rossignac, PhD, ${ }^{\mathrm{d}}$ Mark A. Fogel, MD, ${ }^{\mathrm{e}}$ and Ajit P. Yoganathan, PhD ${ }^{\mathrm{a}}$
}

\begin{abstract}
Background: Using a bifurcated Y-graft as the Fontan baffle is hypothesized to streamline and improve flow dynamics through the total cavopulmonary connection (TCPC). This study conducted numerical simulations to evaluate this hypothesis using postoperative data from 5 patients.
\end{abstract}

\begin{abstract}
Methods: Patients were imaged with cardiac magnetic resonance or computed tomography after receiving a bifurcated aorto-iliac Y-graft as their Fontan conduit. Numerical simulations were performed using in vivo flow rates, as well as 2 levels of simulated exercise. Two TCPC models were virtually created for each patient to serve as the basis for hemodynamic comparison. Comparative metrics included connection flow resistance and inferior vena caval flow distribution.
\end{abstract}

Results: Results demonstrate good hemodynamic outcomes for the Y-graft options. The consistency of inferior vena caval flow distribution was improved over TCPC controls, whereas the connection resistances were generally no different from the TCPC values, except for 1 case in which there was a marked improvement under both resting and exercise conditions. Examination of the connection hemodynamics as they relate to surgical Y-graft implementation identified critical strategies and modifications that are needed to potentially realize the theoretical efficiency of such bifurcated connection designs.

Conclusions: Five consecutive patients received a Y-graft connection to complete their Fontan procedure with positive hemodynamic results. Refining the surgical technique for implementation should result in further energetic improvements that may help improve long-term outcomes. (J Thorac Cardiovasc Surg 2013;145:663-70)

Surgical palliation (the "Fontan procedure") is needed to correct the class of congenital heart defects that result in the presence of a single functional ventricle. The predominant repair of these defects is the total cavopulmonary connection (TCPC), in which the superior vena cava (SVC) and inferior vena cava (IVC) are connected to the pulmonary arteries (PAs). ${ }^{1}$ While representing an energetic improvement over previous designs, the TCPC creates an adverse hemodynamic environment owing to the collision of the

\footnotetext{
From the Wallace H. Coulter Department of Biomedical Engineering, ${ }^{a}$ Georgia Institute of Technology \& Emory University, Atlanta, Ga; the Division of Cardiothoracic Surgery, ${ }^{\text {b }}$ Department of Surgery, Emory University School of Medicine and Children's Healthcare of Atlanta at Egleston, Atlanta, Ga; the Division of Pediatric Cardiology, ${ }^{\mathrm{c}}$ Department of Pediatrics, Emory University School of Medicine and Children's Healthcare of Atlanta at Egleston, Atlanta, Ga; the College of Computing, ${ }^{\mathrm{d}}$ Georgia Institute of Technology, Atlanta, Ga; and the Division of Cardiology, ${ }^{\mathrm{e}}$ Children's Hospital of Philadelphia, Philadelphia, $\mathrm{Pa}$

This study was supported by the National Heart, Lung, and Blood Institute grants HL67622 and R01HL098252 and a Pre-Doctoral Fellowship Award (10PRE3720002) from the American Heart Association.

Disclosures: Authors have nothing to disclose with regard to commercial support.

Received for publication Oct 6, 2011; revisions received Feb 5, 2012; accepted for publication March 12, 2012; available ahead of print May 7, 2012.

Address for reprints: Ajit P. Yoganathan, PhD, Wallace H. Coulter Department of Biomedical Engineering, Georgia Institute of Technology \& Emory University, 313 Ferst Dr, Atlanta, GA 30332 (E-mail: ajit.yoganathan@bme.gatech.edu). $0022-5223 / \$ 36.00$

Copyright (c) 2013 by The American Association for Thoracic Surgery doi:10.1016/j.jtcvs.2012.03.076
}

caval flows. ${ }^{2-4}$ The idea of using a flow bifurcation in the Fontan connection was first proposed by Soerensen and associates 5 ("Optiflo"; US Patent No. 7811244). The motivating concept was that by dividing and rerouting one or both of the vena caval flows, the inefficient collision and mixing at the PA junction can be avoided.

Subsequent studies have analyzed and sought to optimize the design of the Fontan Y-graft (half of the Optiflo) using virtually rendered models. ${ }^{6,7}$ However, the recent work of Kanter and colleagues ${ }^{8}$ represents the first clinical report of Y-graft usage in a consecutive series of Fontan patients. Postoperative imaging data from these patients provide the unique opportunity to evaluate the hemodynamic outcomes of this series and compare them with what could have been realized with possible surgical alternatives. This objective is achieved in this study by numerically evaluating the connections under resting and simulated exercise flow conditions and comparing these performance characteristics with extracardiac TCPC models virtually created for the same patients.

\section{METHODS}

This analysis of clinically collected patient imaging and/or catheterization data was approved by the institutional review boards of Emory University and Georgia Tech in conjunction with an established data sharing agreement between the institutions. Informed consent for the use of image data for research purposes was obtained in each case. 


\section{Abbreviations and Acronyms \\ $\mathrm{CMR}=$ cardiac magnetic resonance \\ HFD = hepatic flow distribution \\ IVC = inferior vena cava \\ LPA = left pulmonary artery \\ $\mathrm{PA}=$ pulmonary artery \\ $\mathrm{PAVM}=$ pulmonary arteriovenous malformation \\ SVC = superior vena cava \\ TCPC $=$ total cavopulmonary connection \\ YCPC $=$ Y-graft cavopulmonary connection}

\section{Patients and Data Acquisition}

Five consecutive patients at Children's Healthcare of Atlanta received a commercially available bifurcated aorto-iliac polytetrafluoroethylene graft (W. L. Gore \& Associates, Inc, Flagstaff, Ariz) as their Fontan baffle. For the purposes of this study, these Y-graft cavopulmonary connections will be referred to as YCPCs. Patient characteristics are provided in Table 1. Cardiac magnetic resonance (CMR) images were acquired for 3 patients before hospital discharge to assess Fontan anatomy and throughplane velocities for selected slices in the IVC, SVC, left PA (LPA), right $\mathrm{PA}$, and aorta (via phase contrast). In 2 cases (1 and 5), computed tomography was instead acquired to provide anatomic information. Preoperative phase contrast CMR data were available for patient 5 to provide flow information, while cardiac catheterization was used to dictate vessel flow rates for patient 1 (see footnote in Table 2). Patient anatomies and (CMR) velocities were reconstructed on the basis of previously published methods. ${ }^{9,10}$ The resulting flow conditions imposed in the numerical simulations are summarized in Table 2.

To provide a basis for hemodynamic comparison to these YCPCs, we made 2 virtual extracardiac TCPC models for each patient representing the alternative connections they may have received instead of a Y-graft. ${ }^{11}$ The 2 designs varied slightly in caval offset and are thus designated as the "t-junction" and "offset" models. Baffle diameters matched the diameter at the base of the Y-graft. Figure 1 shows the anatomic comparison of all connections.

\section{Computational Fluid Dynamics Analysis}

A fully validated, in-house computational solver (based on the sharpinterface immersed boundary method with a fractional step implementation $^{12,13}$ ) was used for all hemodynamic analyses. Cartesian grid spacing was set at $2 \%$ of IVC diameter, which is sufficient for mesh independent results. ${ }^{13}$ The governing equations were solved in their complete unsteady formulation. Time-varying flow conditions (measured from CMR) were imposed in 3 of the patients (2-4) for whom such data were available, while time-averaged conditions were used for the remaining 2 patients. In addition to baseline (resting) flows, simulated exercise conditions were also investigated. Exercise limitations are a known chronic issue with Fontan patients, ${ }^{14,15}$ and previous studies have shown that the nonlinear increases in TCPC power loss with increased flow rates may be an important contributor. ${ }^{16,17}$ Further, inasmuch as small inefficiencies under resting conditions may be amplified and exacerbated with higher flows, these simulations are a means to assess the hemodynamic characteristics and robustness for a given connection geometry. Per standard practice, simulated exercise conditions of 2 and 3 times baseline $(2 \times$ and $3 \times)$ were numerically imposed by respectively doubling and tripling the measured cardiac output and imposing that difference as additional (time-averaged) IVC flow (approximating lower limb exercise).,16 The pulmonary flow splits were maintained constant across these varied conditions as suggested by previous studies. ${ }^{18}$
TABLE 1. Patient characteristics

\begin{tabular}{lccc}
\hline Patient & Age $(\mathbf{y})$ & BSA $\left(\mathbf{m}^{\mathbf{2}}\right)$ & Y-graft size $(\mathbf{m m})$ \\
\hline Patient 1 & 5.4 & 0.72 & $20 \times 10$ \\
Patient 2 & 2.1 & 0.53 & $18 \times 9$ \\
Patient 3 & 3.7 & 0.73 & $20 \times 10$ \\
Patient 4 & 2.3 & 0.47 & $20 \times 10$ \\
Patient 5 & 11.0 & 1.09 & $20 \times 10$ \\
\hline
\end{tabular}

$B S A$, Body surface area.

The primary basis for comparison was the hemodynamic resistance (R) across the connection $\left(R=\frac{\Delta P_{T C P C}}{Q_{V C} / B S A}\right.$; where $\Delta \mathrm{P}$ is pressure drop, $\mathrm{Q}_{\mathrm{VC}}$ is the sum of caval inflows, and BSA is the body surface area). ${ }^{17}$ Additionally, the IVC flow distribution to the PAs, believed to be an important consideration to avoid pulmonary arteriovenous malformations (PAVMs), ${ }^{19}$ was quantified..$^{20}$ These values are reported as "hepatic flow distribution" (HFD). Further, to facilitate qualitative hemodynamic comparisons, the magnitude of viscous dissipation (ie, $\mu\left|\nabla^{2} V\right| ; \mu$ is viscosity, $\mathrm{V}$ is velocity) and 3-dimensional vortex core visualization (via isosurfaces of the second invariant of the deformation matrix, Q) ${ }^{21}$ were also used, where appropriate.

\section{RESULTS}

The resistance and HFD results are detailed in Tables 3 and 4, respectively, and summarized in Figure 2. For resistance, the YCPC group had the lowest average value. However, this trend was skewed by a single case (patient 1 ) for whom there was a significant improvement in efficiency with the YCPC. For the remaining patients, there were only minor differences. The nonlinear response to simulated exercise was consistent among the connections (Figure 2, A).

With respect to HFD under baseline conditions, the Y-grafts had the smallest range and standard deviation among all patients (Figure 2,B). By comparison, the TCPC offset models had a range of almost $100 \%$, denoting significant patient-to-patient variation. Additionally, although there is no accepted "optimal" value for HFD, the mean YCPC value (rest conditions) was closest to $50 \%$, denoting desirable left/right balance.

TABLE 2. Relevant postoperative vessel flow conditions

\begin{tabular}{|c|c|c|c|c|c|c|}
\hline \multirow[b]{2}{*}{ Patient } & \multirow{2}{*}{$\begin{array}{r}\text { CI } \\
\left(\mathbf{L} \cdot \min ^{-1}\right.\end{array}$} & \multirow[b]{2}{*}{$\mathbf{R e}_{\text {IVC }}$} & \multicolumn{4}{|c|}{ Percentage distribution } \\
\hline & & & IVC & SVC (/LSVC) & LPA & RPA \\
\hline Patient $1^{*}$ & 3.8 & 564.0 & 55 & 45 & 48 & 52 \\
\hline Patient 2 & 3.8 & 110.4 & 32 & 68 & 33 & 67 \\
\hline Patient 3 & 3.4 & 178.5 & 45 & 55 & 34 & 66 \\
\hline Patient 4 & 3.0 & 118.2 & 44 & 56 & 74 & 26 \\
\hline Patient 5 & 3.5 & 136.0 & 24 & $55 / 21$ & 68 & 32 \\
\hline
\end{tabular}

$C I$, Cardiac index; $R e_{I V C}$, Reynolds number at the inferior vena cava $(I V C)$ inlet (resting conditions); $S V C$, superior vena cava; $L S V C$, left superior vena cava; $L P A$, left pulmonary artery; $R P A$, right pulmonary artery. *Flow data were taken from cardiac catheterization (cardiac magnetic resonance-measured flows used for all others); pulmonary flow was taken as $\mathrm{Q}_{\mathrm{svC}}$, and difference between pulmonary and systemic flows was taken as $\mathrm{Q}_{\mathrm{IVC}}$. Pulmonary artery split was set as ratio of outlet crosssectional areas. 


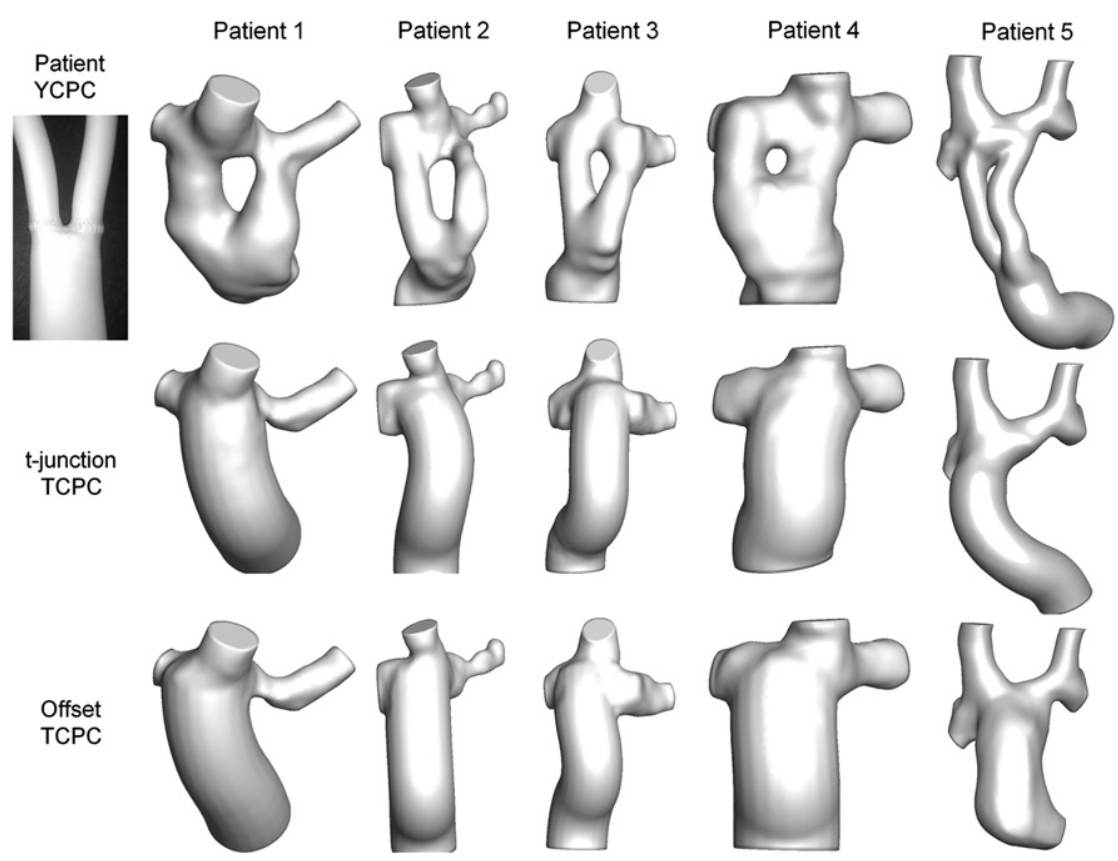

FIGURE 1. Patient YCPCs (top row; with photograph of bifurcated polytetrafluoroethylene graft) and virtually modeled t-junction (middle row) and offset (bottom row) TCPC controls for all 5 patients. YCPC, Y-graft cavopulmonary connection; TCPC, total cavopulmonary connection.

\section{Detailed Hemodynamic Analyses}

As seen in Table 3 and Figure 2, A, the resistance values varied considerably among patients. Thus, rather than focusing on the absolute magnitude of power loss, we seek to identify the factor(s) that mediated energy dissipation differences for a given patient across connection types, to then identify possible means for improvement. Two trends were discernable: dissipation through local vessel constrictions (Figure 3), and dissipation resulting from complex mixing and recirculation (Figure 4).

Figure 3 shows the resting (top) and $3 \times$ (middle) velocity streamlines, as well as resting viscous dissipation contours (bottom) for the YCPC and 1 TCPC option for patients 1, 2, and 5. For patient 1 , there was a section of the LPA that was mildly stenotic, which resulted in significant convective acceleration and dissipation of LPA flow for both connections, even under resting conditions. However, the position of the left branch of the Y-graft helped to augment the size of the vessel, bypass the throat of the constriction, and reduce flow separation in the distal LPA (particularly with exercise), as compared with the t-junction results. These characteristics resulted in a significant reduction of the viscous losses (bottom row).

For patient 2 (Figure 3, B), an acute stenosis in the LPA was again observed, except in this case it was distal to the Y-graft anastomosis. As a result, this patient consistently had among the highest resistance values in the study, irrespective of IVC baffle design (Figure 3, bottom row).

Patient 5 was unique in this series because of the presence of bilateral SVCs and the use of numerical modeling to preoperatively plan the procedure ${ }^{22}$ and suggest surgical revision to a Y-graft. As seen in Figure 5, A, the previous TCPC exhibited severely unbalanced HFD (represented by the blue streamlines) to the PAs (and thus right lung PAVMs developed) because of the unequal distribution of right SVC/left SVC flows (55\% Qs vs $21 \%$ Qs, respectively). In contrast, Figure 5, $B$, shows that the Y-graft delivers IVC flow to both PAs. With respect to energy

TABLE 3. Connection resistance summary $\left(\mathrm{mm} \mathrm{Hg} \cdot \min ^{-1} \cdot \mathrm{L}^{-1}\right)$ at rest and exercise

\begin{tabular}{|c|c|c|c|c|c|c|c|c|c|}
\hline \multirow[b]{2}{*}{ Patient } & \multicolumn{3}{|c|}{ Rest } & \multicolumn{3}{|c|}{$2 \times$} & \multicolumn{3}{|c|}{$3 \times$} \\
\hline & YСРC & t-Junction & Offset & YCPC & t-Junction & Offset & YCPC & t-Junction & Offset \\
\hline Patient 1 & 0.33 & 1.26 & 1.21 & 0.74 & 2.57 & 2.29 & 1.22 & 3.95 & 3.43 \\
\hline Patient 2 & 1.6 & 1.6 & 1.5 & 2.63 & 2.42 & 2.60 & 3.93 & 3.83 & 3.83 \\
\hline Patient 3 & 0.17 & 0.15 & 0.23 & 0.40 & 0.32 & 0.46 & 0.68 & 0.51 & 0.62 \\
\hline Patient 4 & 0.2 & 0.19 & 0.19 & 0.57 & 0.55 & 0.67 & 0.89 & 0.90 & 1.08 \\
\hline Patient 5 & 0.33 & 0.33 & 0.32 & 1.80 & 1.50 & 1.62 & 3.56 & 2.54 & 2.81 \\
\hline Mean & 0.53 & 0.71 & 0.69 & 1.23 & 1.47 & 1.53 & 2.06 & 2.35 & 2.35 \\
\hline
\end{tabular}

$Y C P C$, Y-graft cavopulmonary connection. 
TABLE 4. HFD results (\%LPA)

\begin{tabular}{|c|c|c|c|c|c|c|c|c|c|}
\hline \multirow[b]{2}{*}{ Patient } & \multicolumn{3}{|c|}{ Rest } & \multicolumn{3}{|c|}{$2 \times$} & \multicolumn{3}{|c|}{$3 \times$} \\
\hline & YСРC & t-junction & Offset & YCPC & t-junction & Offset & YCPC & t-junction & Offset \\
\hline Patient 1 & 70 & 65 & 42 & 51 & 56 & 38 & 47 & 52 & 42 \\
\hline Patient 2 & 32 & 16 & 4 & 30 & 14 & 9 & 30 & 24 & 20 \\
\hline Patient 3 & 49 & 57 & 28 & 45 & 47 & 42 & 44 & 43 & 41 \\
\hline Patient 4 & 52 & 46 & 35 & 58 & 56 & 60 & 66 & 65 & 69 \\
\hline Patient 5 & 49 & 31 & 100 & 58 & 56 & 82 & 62 & 60 & 76 \\
\hline Mean & 51 & 43 & 42 & 49 & 46 & 46 & 50 & 49 & 49 \\
\hline SD & 14 & 20 & 36 & 12 & 18 & 27 & 15 & 16 & 23 \\
\hline
\end{tabular}

$H F D$, Hepatic flow distribution; $L P A$, left pulmonary artery; $Y C P C$, Y-graft cavopulmonary connection; $S D$, standard deviation.

efficiency, however, the extended length of the Y-graft conduit (Figure 1) caused significant acceleration and flow separation through the branches (Figure 3,C) that increased energy losses.

For the remaining 2 patients (3, top; 4, bottom), Figure 4 shows velocity streamlines $(A)$ and coherent vortex cores $(B$; to facilitate appreciation of the complex flow recirculation). In these patients, both branches of the Y-graft were connected nearly perpendicularly to the PAs, resulting in significant flow recirculation at the anastomoses (arrow, Figure 4, A). The vortex cores (Figure 4, B) provide improved visualization of these rotational flow structures and show their expansive size. Rather than being local, contained phenomena, these vortices bilaterally originated in the high shear wake region distal to the IVC bifurcation and extended downstream into the PAs. As a result, Figure 4, $C$, shows that local regions of relatively high energy dissipation (from the color contours) were created at multiple downstream locations adjacent to these cores because of the complex fluid motion.

\section{DISCUSSION}

This study, in conjunction with the clinical report of Kanter and associates, ${ }^{8}$ clearly demonstrates that the surgical implementation of the Fontan Y-graft is feasible. The additional contribution of this work is the identification and elucidation of the hemodynamic implications associated with the characteristics of the surgical design. In other words, the use of a Y-graft does not necessarily imply that it is as optimally efficient as in idealized studies. ${ }^{5-7}$ The following sections will analyze the numerically derived 3-dimensional velocity fields in the context of the causative geometric design characteristics.

\section{Hemodynamic Efficiency}

The theoretical benefits of flow bifurcation in the cavopulmonary pathway originated with the experimental and numerical work of Soerensen and associates, ${ }^{5}$ in which the multiple bifurcations of the Optiflo were shown to outperform an idealized TCPC model. Rather than requiring a collision and $90^{\circ}$ redirection of caval flows, the Optiflo efficiently redirected flow, ensuring a smooth and streamlined transition to the PAs. Marsden and associates ${ }^{7}$ followed with a numerical investigation in patient-specific models, focusing only on the bifurcation of the IVC, demonstrating again the potential for energetic improvements.

These hypothetical gains were not realized in the present in vivo implementations. In 4 of the 5 cases, Y-graft
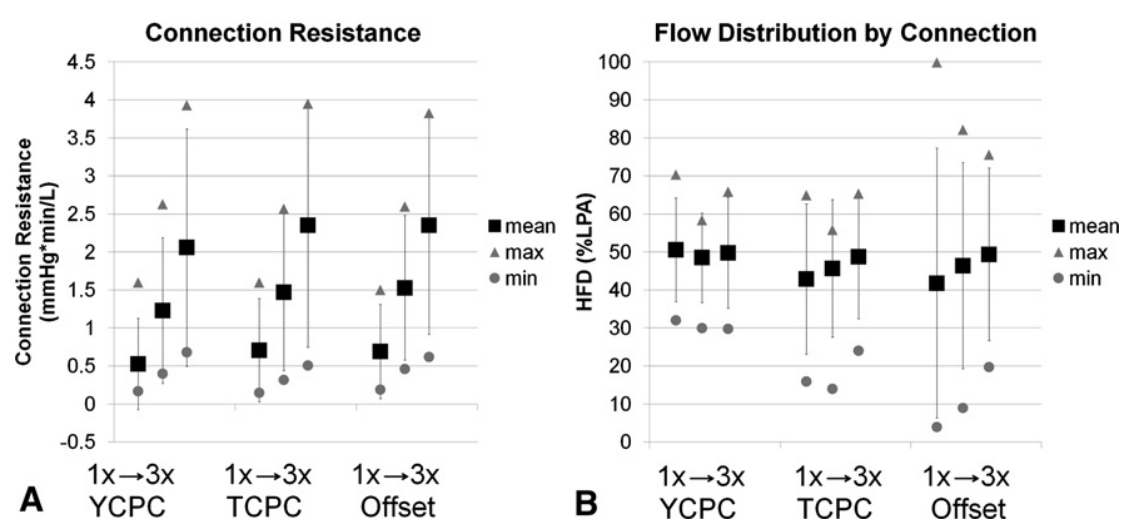

FIGURE 2. Mean, maximum, and minimum values of connection resistance (A) and flow distribution (B) for each connection (YCPC, TCPC, offset) and flow condition $(1 \times, 2 \times, 3 \times)$ investigated. $Y C P C$, Y-graft cavopulmonary connection; $T C P C$, total cavopulmonary connection; HFD, hepatic flow distribution; $L P A$, left pulmonary artery. 


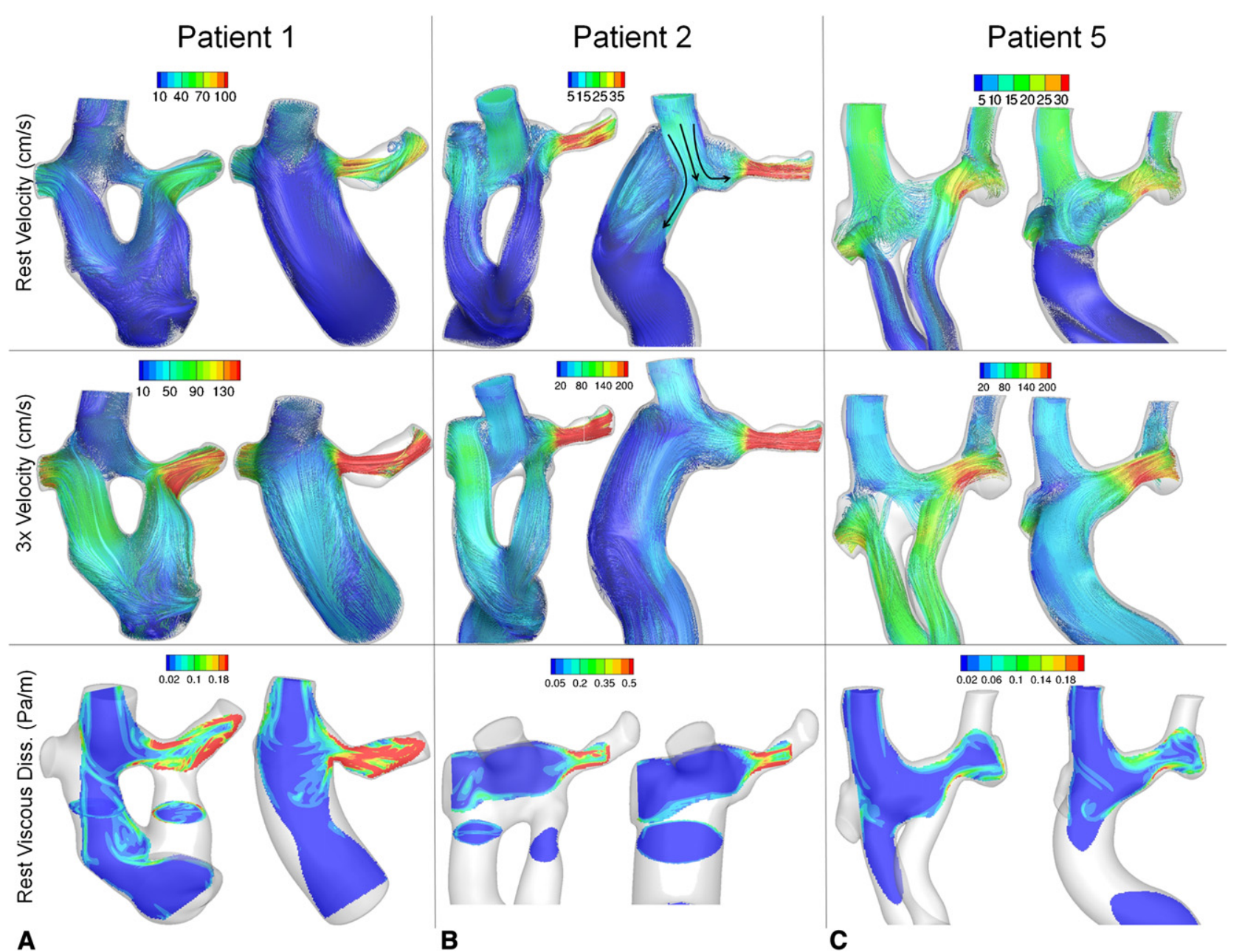

FIGURE 3. Comparison of YCPC and TCPC hemodynamics for patients 1 (A), 2 (B; representative image), and 5 (C). The resting velocity streamlines (top row), $3 \times$ velocity streamlines (middle row), and viscous dissipation contours (bottom row) are shown in each case. YCPC, Y-graft cavopulmonary connection; $T C P C$, total cavopulmonary connection.

resistance was approximately equal to the resistances of the virtual TCPC controls. Clearly, since this is the first attempt at systematic Y-graft use for the Fontan procedure, a learning curve to optimize the surgical implementation should be anticipated. In that regard, it is promising that there is no evidence in this series of diminished early outcome. These results suggest that the YCPC may be "forgiving" enough to still be energetically equivalent to the TCPC when the design is theoretically suboptimal. Yet, there is also clear evidence from at least 1 case that the energetics can be better than the TCPC.

In that case (patient 1), the decreased resistance is attributable to the fact that the Y-graft position augmented and bypassed an acute stenosis in the LPA that was otherwise a significant resistive element. Given the recent report showing the prevalence of subaortic PA stenosis ${ }^{23}$ in single ventricle patients (particularly those with hypoplastic left heart syndrome), this potential strength of the Y-graft is something to consider for targeted use. However, this specific application does not tap into the fundamental characteristics of the Y-graft that make it efficient.

One possible reason for the efficiency differences is the size selected for the Y-graft branches. Larger branches allow for lower fluid velocities and shear stresses ${ }^{7}$; however, they also impose increased spatial constraints and are not available "off the shelf," as the current grafts are. Without a prefabricated graft, the bifurcation must be constructed ad hoc, which creates an additional set of design variables and possible failure modes (eg, thrombosis formation along additional suture lines). As such, a more pragmatic question to ask is, can the efficiency results be improved with the existing graft design to remove the need for pursuing size and construction alternatives?

A recent study by Yang and coworkers ${ }^{6}$ sought to parametrically optimize the Y-graft design (eg, branch placement, angle, size). While, in theory, such an analysis would be helpful in directing surgical decision making, insufficient mathematical constraint of the design variables 


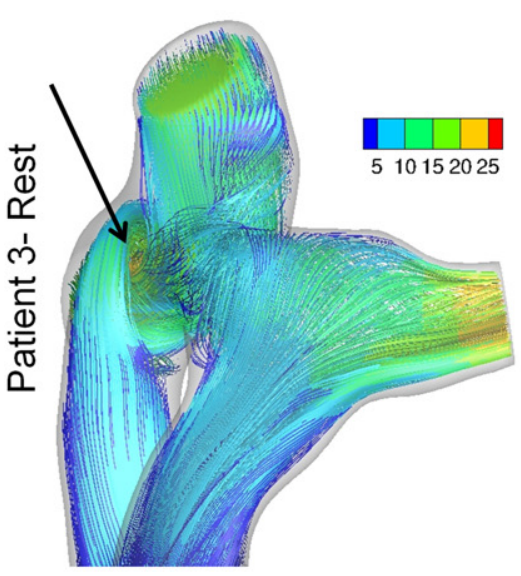

Velocity $(\mathrm{cm} / \mathrm{s})$



A

B

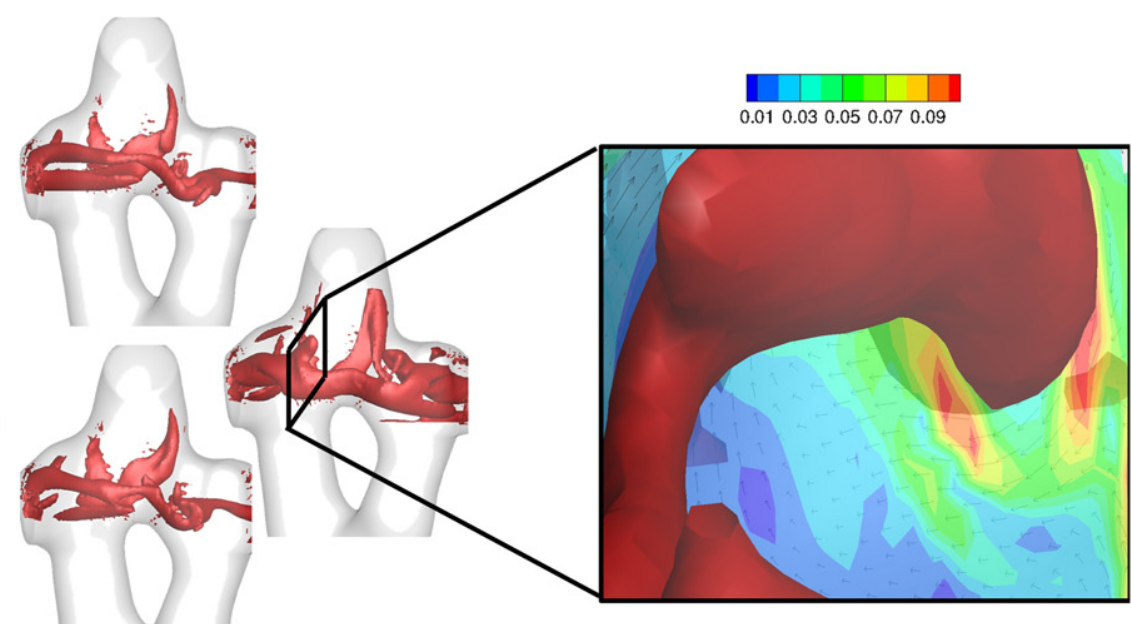

Vortex Core Isosurfaces

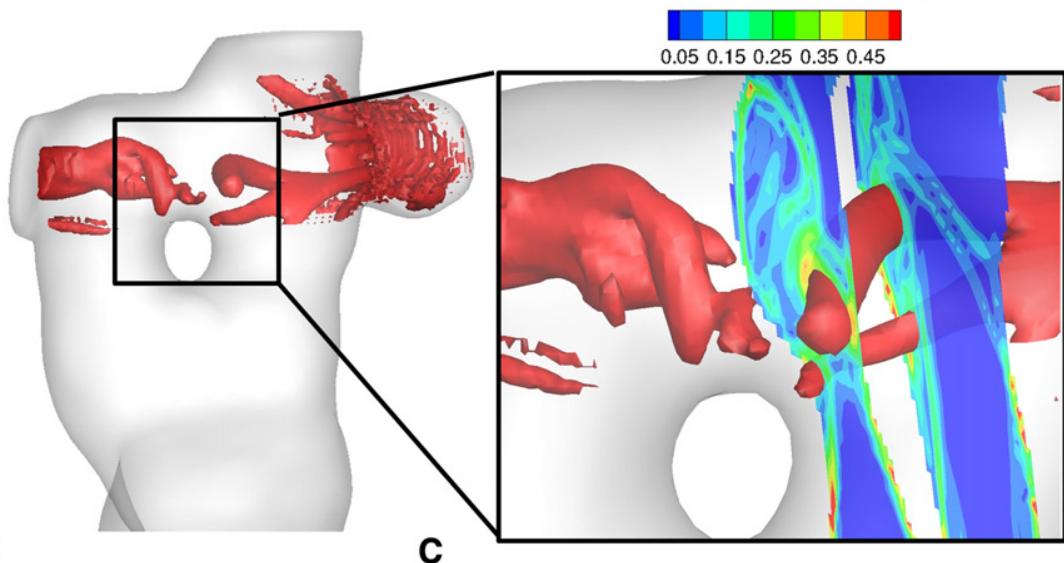

C

FIGURE 4. Velocity streamlines (A), vortex core isosurfaces $(\mathrm{Q}=800$ [top], 13,000 [bottom]) (B), and viscous dissipation contours localized to the vortex isosurfaces (C) for patients 3 (top, rest) and 4 (bottom, $3 \times$ ).

limits the ability to translate those results into quantitative guidelines for surgical implementation. However, the demonstrated importance of tangential alignment of the bifurcation branches with the PA axis is a relevant message that was implicit in the original Optiflo design concept and provides an interesting perspective for the present results.

In this light, there were 2 primary shortcomings in these patient connections that limited hemodynamic efficiency and should be surgically addressed in future cases. The first was the near perpendicular angle of approach in most of the connections. This perpendicular connection creates a scenario in which the IVC flow enters the PA, impinges on the opposite (eg, superior) vessel wall, and must undergo significant secondary recirculation to align with the PA axis of flow. As shown in Figure 4, this recirculation may create dissipative secondary flow structures that can extend to significant lengths downstream and decrease overall connection efficiency. Introducing an anterior curvature to the baffle may facilitate better angulation than a straight $\mathrm{t}$-junction approach would allow. Beveling the distal ends of the graft is also suggested to achieve the efficiency benefits of a flared connection. ${ }^{24}$

The second shortcoming was the close proximity or even medial (to the SVC) placement of the Y-graft branches, which allows for continued interaction and mixing between the caval flows. In the extreme case in which 1 branch was positioned exactly opposite the SVC (as in patients 1 [Figure 3,A] and 4 [Figure 4, bottom]), there is direct caval flow competition and the associated energy dissipation that a bifurcated design was intended to avoid. Placing the right branch distal to the right pulmonary upper lobe and the left branch immediately posterior to the ascending aorta provides the best means to avoid that scenario. Furthermore, positioning of the SVC at the Glenn stage could be adjusted in future Y-graft candidates to provide additional lateral space for the branches.

Importantly, these design factors are independent of graft size and, in fact, smaller branches would facilitate placement according to these guidelines. This provides additional support for the present choice in Y-graft design and 

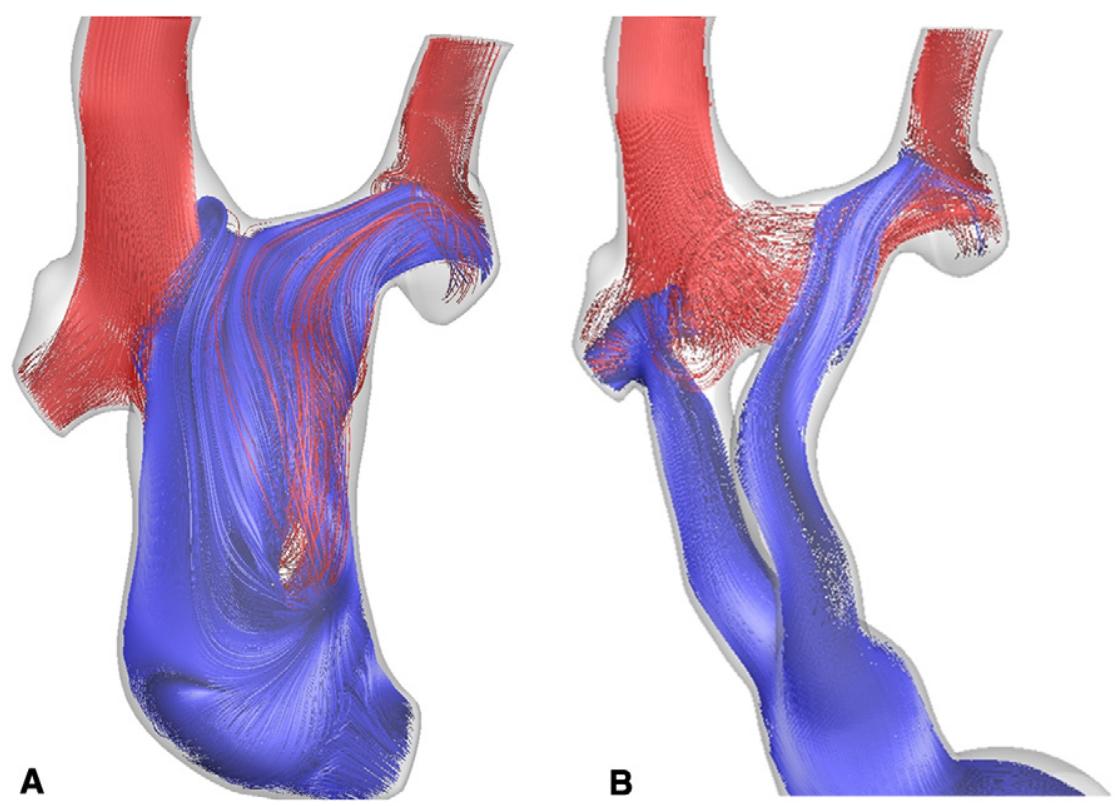

FIGURE 5. Streamlines color coded by vessel of origin ( red, superior vena cava; blue, inferior vena cava) for (A) the patient 5 offset TCPC (ie, the failed connection before surgical revision) and (B) the postrevision YCPC. YCPC, Y-graft cavopulmonary connection; TCPC, total cavopulmonary connection.

indicates that future studies should pursue these design optimization modifications before ultimately answering the Y-graft sizing question.

With regard to the originally posed hypothesis, this work shows that the Fontan Y-graft can be effectively used in certain scenarios. Yet, this fact neither proves nor disproves the hypothesis with respect to energy efficiency because the question remains: can the suggested modifications ensure that the "optimal" Fontan Y-graft is consistently realized under anatomic size constraints? The answer is likely to be patient-specific in that the position and size of the PA, pulmonary veins, SVC, and aorta are sure to be the critical determinants of whether or not the desired shape and position of the Y-graft is achievable. Herein lies a potential application for patient-specific simulation-based surgical planning techniques for Fontan surgery, ${ }^{22,25}$ which could be used to both select candidates for favorable YCPC implementation and visually provide the target landmarks for branch placement.

\section{Caval Flow Distribution}

Balanced HFD is an important consideration to prevent the formation of PAVM ${ }^{19}$ There is a lack of evidence to indicate the minimum thresholds to prevent PAVM formation; however, a split at or around 50:50 is a desirable target. In considering the HFD values of the various connections evaluated in this series, there was no clinical significance in the mean differences (YCPC vs t-junction vs offset), as $40 \%$ versus $50 \%$ HFD would not affect the patient outcomes. However, the smaller variance across the YCPC results is noteworthy because it demonstrates improved consistency and stability of that design. By comparison, there were 3 instances with TCPCs that exhibited severely unbalanced HFD. So while, on average, the Y-graft may not significantly improve IVC flow distributions, these data suggest it could reduce the occurrence of outliers with poor streaming performance. Such outliers are far more prevalent in specific groups, such as cases of interrupted IVC, ${ }^{26}$ suggesting that these are additional patients who may benefit from targeted Y-graft use over extracardiac baffles. $^{22,25}$

\section{Limitations}

The primary limitations of this study are the assumption of rigid walls and the use of time-averaged boundary conditions for many of the simulations. Although such assumptions have a slight effect on the quantitative output of the simulations, they would have affected the various geometries similarly and are therefore unlikely to alter the comparative conclusions drawn. In addition, there is obviously a lack of long-term clinical follow-up for this patient series to determine the implications of the simulated hemodynamics, yet the results are sufficiently encouraging to motivate such long-term follow-up. This is the first hemodynamic evaluation of the in vivo Fontan Y-graft and, therefore, despite these limitations, there is still significant clinical value to be gained from the results presented.

\section{CONCLUSIONS}

In summary, the detailed hemodynamics of the Fontan Y-graft in an in vivo setting have been investigated for the first time under a variety of relevant flow conditions and 
with direct comparison with patient-specific TCPC controls. Desirable IVC-PA flow distribution characteristics were found; however, the flow efficiency performance fell short of the outcomes predicted by previous models. A beneficial comparison was found when the Y-graft was able to offset the effects of acute PA stenosis, making such situations a potential for targeted usage. Further, detailed analysis of the surgical Y-graft implementation with respect to the resulting flow fields was used to identify strategies for improving performance on a broader scale. Future studies will model these hypothesized improvements and characterize the performance gains to assess surgical feasibility of the "optimal" Y-graft design.

\section{References}

1. de Leval MR, Kilner P, Gewillig M, Bull C. Total cavopulmonary connection: a logical alternative to atriopulmonary connection for complex Fontan operations. J Thorac Cardiovasc Surg. 1988;96:682-95.

2. Sharma S, Goudy S, Walker P, Panchal S, Ensley A, Kanter K, et al. In vitro flow experiments for determination of optimal geometry of total cavopulmonary connection for surgical repair of children with functional single ventricle. J Am Coll Cardiol. 1996;27:1264-9.

3. Marsden AL, Vignon-Clementel IE, Chan FP, Feinstein JA, Taylor CA. Effects of exercise and respiration on hemodynamic efficiency in CFD simulations of the total cavopulmonary connection. Ann Biomed Eng. 2007;35: 250-63.

4. de Leval MR, Dubini G, Migliavacca F, Jalali H, Camporini G, Redington A, et al. Use of computational fluid dynamics in the design of surgical procedures: application to the study of competitive flows in cavopulmonary connections. J Thorac Cardiovasc Surg. 1996;111:502-13.

5. Soerensen DD, Pekkan K, de Zelicourt D, Sharma S, Kanter K, Fogel M, et al. Introduction of a new optimized total cavopulmonary connection. Ann Thorac Surg. 2007;83:2182-90.

6. Yang W, Feinstein JA, Marsden AL. Constrained optimization of an idealized Y-shaped baffle for the Fontan surgery at rest and exercise. Comput Meth Appl Mech Eng. 2010;199:2135-49.

7. Marsden AL, Bernstein AJ, Reddy VM, Shadden SC, Spilker RL, Chan FP, et al. Evaluation of a novel Y-shaped extracardiac Fontan baffle using computational fluid dynamics. J Thorac Cardiovasc Surg. 2009;137:394-403.

8. Kanter KR, Haggerty CM, Restrepo M, de Zelicourt D, Rossignac J, Parks WJ, et al. Preliminary clinical experience with a bifurcated Y-graft Fontan procedure (abstract). Presented at: The Western Thoracic Surgical Association 37th Annual Meeting; June 22-25, 2011; Colorado Springs, CO.

9. Frakes DH, Conrad CP, Healy TM, Monaco JW, Fogel M, Sharma S, et al. Application of an adaptive control grid interpolation technique to morphological vascular reconstruction. IEEE Trans Biomed Eng. 2003;50:197-206.

10. Sundareswaran K, Frakes D, Fogel M, Soerensen D, Oshinski JN, Yoganathan A. Optimum fuzzy filters for phase-contrast magnetic resonance imaging segmentation. J Magn Reson Imaging. 2009;29:155-65.
11. Pekkan K, Whited B, Kanter K, Sharma S, de Zelicourt D, Sundareswaran K, et al. Patient-specific surgical planning and hemodynamic computational fluid dynamics optimization through free-form haptic anatomy editing tool (SURGEM). Med Biol Eng Comput. 2008;46:1139-52.

12. Ge L, Sotiropoulos F. A numerical method for solving the $3 \mathrm{~d}$ unsteady incompressible navier-stokes equations in curvilinear domains with complex immersed boundaries. J Comput Phys. 2007;225:1782-809.

13. de Zélicourt D, Ge L, Wang C, Sotiropoulos F, Gilmanov A, Yoganathan AP. Flow simulations in arbitrarily complex cardiovascular anatomies-an unstructured Cartesian grid approach. Comput Fluids. 2009;38:1749-62.

14. Rosenthal M, Bush A, Deanfield J, Redington A. Comparison of cardiopulmonary adaptation during exercise in children after the atriopulmonary and total cavopulmonary connection Fontan procedures. Circulation. 1995;91: 372-8.

15. Shachar G, Fuhrman B, Wang Y, Lucas RJ, Lock J. Rest and exercise hemodynamics after the Fontan procedure. Circulation. 1982;65:1043-8.

16. Whitehead KK, Pekkan K, Kitajima HD, Paridon SM, Yoganathan AP, Fogel MA. Nonlinear power loss during exercise in single-ventricle patients after the Fontan: insights from computational fluid dynamics. Circulation. 2007; 116(11 Suppl):I165-71.

17. Sundareswaran KS, Pekkan K, Dasi LP, Whitehead K, Sharma S, Kanter K, et al. The total cavopulmonary connection resistance: a significant impact on single ventricle hemodynamics at rest and exercise. Am J Physiol Heart Circ Physiol. 2008;295:H2427-35.

18. Pedersen EM, Stenbog EV, Frund T, Houlind K, Kromann O, Sorensen KE, et al. Flow during exercise in the total cavopulmonary connection measured by magnetic resonance velocity mapping. Heart. 2002;87:554-8.

19. Duncan B, Desai S. Pulmonary arteriovenous malformations after cavopulmonary anastomosis. Ann Thorac Surg. 2003;76:1759-66.

20. Dasi LP, Whitehead K, Pekkan K, de Zelicourt D, Sundareswaran K, Kanter K, et al. Pulmonary hepatic flow distribution in total cavopulmonary connections: extracardiac versus intracardiac. J Thorac Cardiovasc Surg. 2011;141:207-14.

21. Hunt JCR, Wray AA, Moin P. Eddies, stream, and convergence zones in turbulent flows. Center for Turbulence Research Report CTR-S88. 1988;193.

22. De Zelicourt D, Haggerty CM, Sundareswaran KS, Whited B, Rossignac J, Kanter K, et al. Individualized computer-based surgical planning to address pulmonary ateriovenous malformations in patients with a single ventricle with an interrupted inferior vena cava and azygous continuation. $J$ Thorac Cardiovasc Surg. 2011;141:1170-7.

23. Dasi LP, Sundareswaran KS, Sherwin C, de Zelicourt D, Kanter KR, Fogel MA, et al. Larger aortic reconstruction corresponds to diminished left pulmonary artery size in patients with single ventricle phyisology. J Thorac Cardiovasc Surg. 2010;139:557-61.

24. Ensley A, Lynch P, Chatzimavroudis GP, Lucas CL, Sharma S, Yoganathan AP. Toward designing the optimal total cavopulmonary connection: an in vitro study. Ann Thorac Surg. 1999;68:1384-90.

25. Sundareswaran K, de Zélicourt D, Sharma S, Kanter K, Spray T, Rossignac JR, et al. Correction of pulmonary arteriovenous malformation using image based surgical planning. JACC Imag. 2009;2:1024-30.

26. McElhinney DB, Marx GR, Marshall AC, Mayer JEJ, del Nido P. Cavopulmonary pathway modification in patients with heterotaxy and newly diagnosed or persistent pulmonary arteriovenous malformations after a modified Fontan operation. J Thorac Cardiovasc Surg. 2011;131:1362-70. 